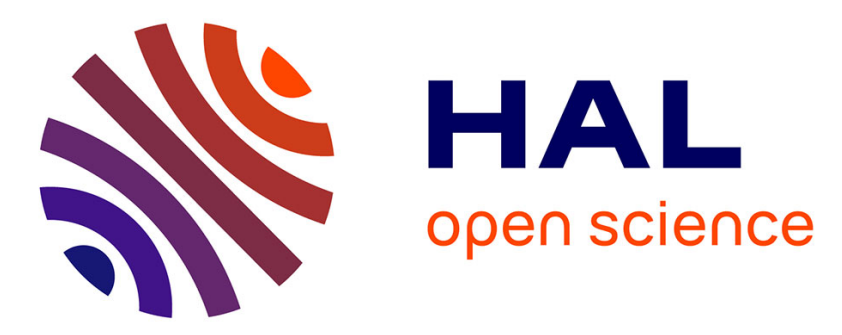

\title{
Minimum density of identifying codes of king grids
}

Rennan Dantas, Rudini Sampaio, Frédéric Havet

\section{To cite this version:}

Rennan Dantas, Rudini Sampaio, Frédéric Havet. Minimum density of identifying codes of king grids. Electronic Notes in Discrete Mathematics, 2017, 62, pp.51 - 56. 10.1016/j.endm.2017.10.010 . hal01634305

\section{HAL Id: hal-01634305 \\ https://hal.inria.fr/hal-01634305}

Submitted on 13 Nov 2017

HAL is a multi-disciplinary open access archive for the deposit and dissemination of scientific research documents, whether they are published or not. The documents may come from teaching and research institutions in France or abroad, or from public or private research centers.
L'archive ouverte pluridisciplinaire HAL, est destinée au dépôt et à la diffusion de documents scientifiques de niveau recherche, publiés ou non, émanant des établissements d'enseignement et de recherche français ou étrangers, des laboratoires publics ou privés. 


\title{
Minimum density of identifying codes of king grids
}

\author{
Rennan Dantas, Rudini M. Sampaio ${ }^{1}$ \\ Universidade Federal do Ceará, Fortaleza, Brazil \\ Frédéric Havet ${ }^{1}$ \\ Université Côte d'Azur, CNRS, I3S, INRIA, France
}

\begin{abstract}
A set $C \subseteq V(G)$ is an identifying code in a graph $G$ if for all $v \in V(G), C[v] \neq \emptyset$, and for all distinct $u, v \in V(G), C[u] \neq C[v]$, where $C[v]=N[v] \cap C$ and $N[v]$ denotes the closed neighbourhood of $v$ in $G$. The minimum density of an identifying code in $G$ is denoted by $d^{*}(G)$. In this paper, we study the density of king grids which are strong product of two paths. We show that for every king grid $G, d^{*}(G) \geq 2 / 9$. In addition, we show this bound is attained only for king grids which are strong products of two infinite paths. Given $k \geq 3$, we denote by $\mathcal{K}_{k}$ the (infinite) king strip with $k$ rows. We prove that $d^{*}\left(\mathcal{K}_{3}\right)=1 / 3, d^{*}\left(\mathcal{K}_{4}\right)=5 / 16, d^{*}\left(\mathcal{K}_{5}\right)=4 / 15$ and $d^{*}\left(\mathcal{K}_{6}\right)=5 / 18$. We also prove that $\frac{2}{9}+\frac{8}{81 k} \leq d^{*}\left(\mathcal{K}_{k}\right) \leq \frac{2}{9}+\frac{4}{9 k}$ for every $k \geq 7$.
\end{abstract}

Keywords: Identifying code, King grid, Discharging Method.

\section{Introduction}

Let $G$ be a graph. The neighbourhood of a vertex $v$ of $G$, denoted by $N(v)$, is the set of vertices adjacent to $v$ in $G$, and the closed neighbourhood of $v$ is the set $N[v]=N(v) \cup\{v\}$. Given a set $C \subseteq V(G)$, let $C[v]=N[v] \cap C$. We say that $C$ is an identifying code of $G$ if $C[v] \neq \emptyset$ for all $v \in V(G)$, and $C[u] \neq C[v]$ for all distinct $u, v \in V(G)$. Clearly, a graph $G$ has an identifying code if and only if it contains no twins (vertices $u, v \in V(G)$ with $N[u]=N[v]$ ).

Let $G$ be a (finite or infinite) graph with bounded maximum degree. For any non-negative integer $r$ and vertex $v$, we denote by $B_{r}(v)$ the ball of radius $r$ in $G$ centered at $v$, that is $B_{r}(v)=\{x \mid \operatorname{dist}(v, x) \leq r\}$. For any set of vertices $C \subseteq V(G)$, the density of $C$ in $G$, denoted by $d(C, G)$, is defined by

$$
d(C, G)=\limsup _{r \rightarrow+\infty} \frac{\left|C \cap B_{r}\left(v_{0}\right)\right|}{\left|B_{r}\left(v_{0}\right)\right|}
$$

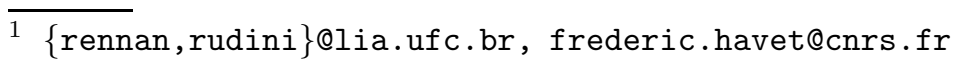


where $v_{0}$ is an arbitrary vertex in $G$. The infimum of the density of an identifying code in $G$ is denoted by $d^{*}(G)$. Observe that if $G$ is finite, then $d^{*}(G)=\left|C^{*}\right| /|V(G)|$, where $C^{*}$ is a minimum-size identifying code of $G$.

The problem of finding low-density identifying codes was introduced in [12] in relation to fault diagnosis in arrays of processors. Particular interest was dedicated to grids as many processor networks have a grid topology. Many results have been obtained on square grids $[4,1,9,2,11]$, triangular grids $[12,10]$, and hexagonal grids $[5,7,8]$. In this paper, we study king grids, which are strong products of two paths. The strong product of two graphs $G$ and $H$, denoted by $G \otimes H$, is the graph with vertex set $V(G) \times V(H)$ and edge set:

$$
\begin{aligned}
E(G \otimes H)= & \left\{(a, b)\left(a, b^{\prime}\right) \mid a \in V(G) \text { and } b b^{\prime} \in E(H)\right\} \\
& \cup\left\{(a, b)\left(a^{\prime}, b\right) \mid a a^{\prime} \in E(G) \text { and } b \in V(H)\right\} \\
& \cup\left\{(a, b)\left(a^{\prime}, b^{\prime}\right) \mid a a^{\prime} \in E(G) \text { and } b b^{\prime} \in E(H)\right\} .
\end{aligned}
$$

The two-way infinite path, denoted by $P_{\mathbb{Z}}$, is the graph with vertex set $\mathbb{Z}$ and edge set $\{\{i, i+1\} \mid \in \mathbb{Z}\}$, and the one-way infinite path, denoted by $P_{\mathbb{N}}$, is the graph with vertex set $\mathbb{N}$ and edge set $\{\{i, i+1\} \mid i \in \mathbb{N}\}$. A path is a connected subgraph of $P_{\mathbb{Z}}$. For every positive integer $k, P_{k}$ is the subgraph of $P_{\mathbb{Z}}$ induced by $\{1,2, \ldots, k\}$. A king grid is the strong product of two (finite or infinite) paths. The plane king grid is $\mathcal{G}_{K}=P_{\mathbb{Z}} \otimes P_{\mathbb{Z}}$, the half-plane king grid is $\mathcal{H}_{K}=P_{\mathbb{Z}} \otimes P_{\mathbb{N}}$, the quater-plane king grid is $\mathcal{Q}_{K}=P_{\mathbb{N}} \otimes P_{\mathbb{N}}$, and the king strip of height $k$ is $\mathcal{K}_{k}=P_{\mathbb{Z}} \otimes P_{k}$.

In 2002, Charon et al. [3] proved that $d^{*}\left(\mathcal{G}_{K}\right)$ is $2 / 9$. They provided the tile depicted in Figure 1, which generates a periodic tiling of the plane with periods $(0,6)$ and $(6,0)$, yielding an identifying code $C_{\infty}$ of the bidimensional infinite king grid with density $\frac{2}{9}$.

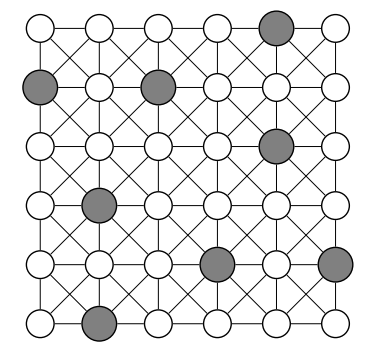

Fig. 1. Tile generating an optimal identifying code of the bidimensional infinite grid. Black vertices are those of the code.

In this paper, using the Discharging Method (see Section 3 of [10] for a detailed presentation of this technique for identifying codes), we provide the following tight general lower bound on the minimum density of identifying 
codes of king grids.

Theorem 1.1 If $G$ is a (finite or infinite) king grid, then $d^{*}(G) \geq \frac{2}{9}$.

Keeping on, we prove the following.

Theorem 1.2 If $G$ is a finite king grid, then $d^{*}(G)>\frac{2}{9}$.

Finally, we give some bounds for king strips. Pushing further the proof of Theorem 1.1, we prove the following.

Theorem 1.3 For every $k \geq 6, d^{*}\left(\mathcal{K}_{k}\right) \geq \frac{2}{9}+\frac{8}{81 k}$.

Modifying $C_{\infty}$, we construct identifying codes of $\mathcal{K}_{k}$ yielding the following upper bounds.

Theorem 1.4 For every $k \geq 5$,

$$
d^{*}\left(\mathcal{K}_{k}\right) \leq\left\{\begin{array}{lll}
\frac{2}{9}+\frac{6}{18 k}, & \text { if } k \equiv 0 & \bmod 3, \\
\frac{2}{9}+\frac{8}{18 k}, & \text { if } k \equiv 1 & \bmod 3 \\
\frac{2}{9}+\frac{7}{18 k}, & \text { if } k \equiv 2 & \bmod 3
\end{array}\right.
$$

Finally, we show some identifying codes of $\mathcal{K}_{3}, \mathcal{K}_{4}, \mathcal{K}_{5}$ and $\mathcal{K}_{6}$ (see Figures $2,3,4$, and 5.) and prove that they are optimal. This yields the following.

Theorem $1.5 d^{*}\left(\mathcal{K}_{3}\right)=1 / 3=0.333 \ldots \quad d^{*}\left(\mathcal{K}_{4}\right)=5 / 16=0.3125$

$$
d^{*}\left(\mathcal{K}_{5}\right)=4 / 15=0.2666 \ldots \quad d^{*}\left(\mathcal{K}_{6}\right)=5 / 18=0.2777 \ldots
$$

Clearly $d^{*}\left(\mathcal{K}_{1}\right)=1 / 2\left(\right.$ as $\left.\mathcal{K}_{1}=\mathcal{S}_{1}=P_{\mathbb{Z}}\right)$ and $\mathcal{K}_{2}$ has no identifying code because it has twins. All these results imply that $\mathcal{G}_{K}, \mathcal{H}_{K}$ and $\mathcal{Q}_{K}$ are the unique king grids having an identifying code with density $2 / 9$. (One can easily derive from $C_{\infty}$ identifying codes with density $2 / 9$ of $\mathcal{H}_{K}$ and $\mathcal{Q}_{K}$ ).
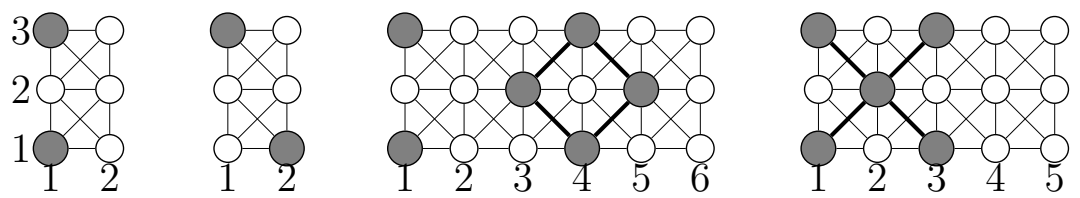

Fig. 2. Four tiles generating optimal identifying codes of $\mathcal{K}_{3}$ (density $1 / 3$ )

\section{Sketches of proofs}

Sketch of proof of Theorem 1.1. Let $G$ be a king grid and $C$ an identifying code of $G$. We shall prove that $d(C, G) \geq 2 / 9$. For this, we use the Discharging 


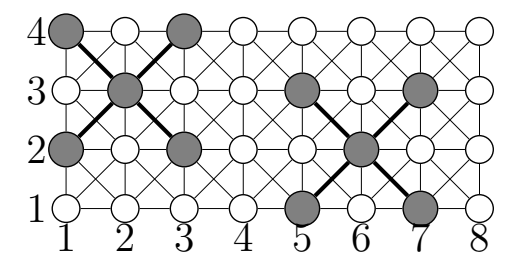

Fig. 3. Tile generating an optimal identifying code of $\mathcal{K}_{4}$ (density $5 / 16$ )
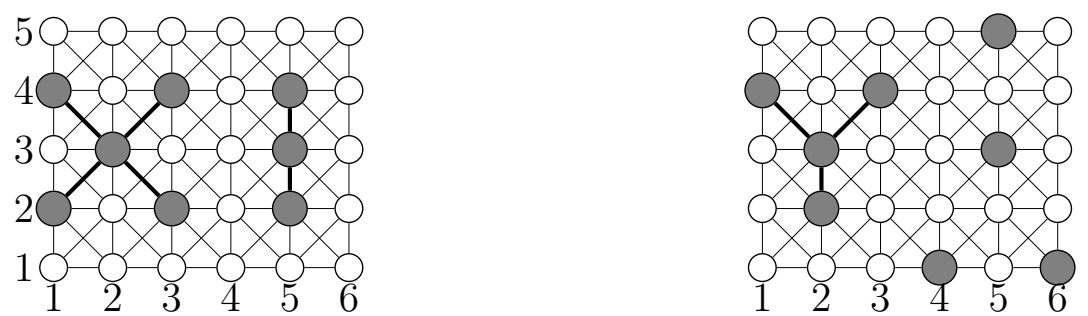

Fig. 4. Two tiles generating optimal identifying codes of $\mathcal{K}_{5}$ (density $4 / 15$ )
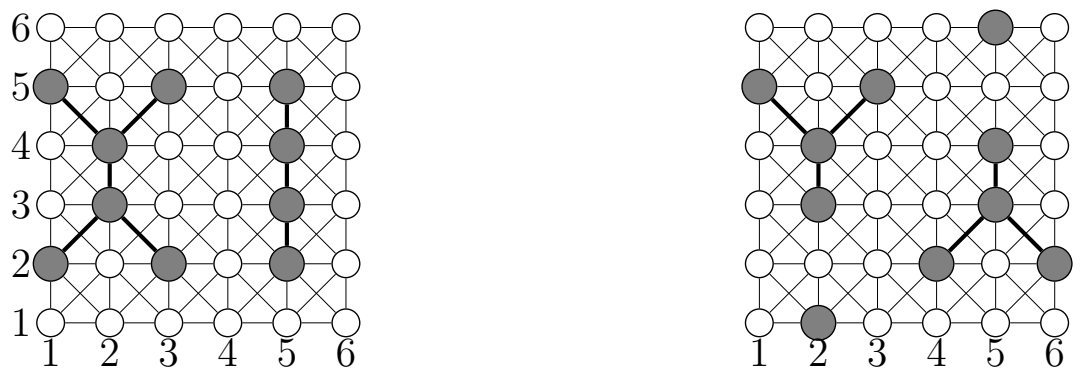

Fig. 5. Two tiles generating optimal identifying codes of $\mathcal{K}_{6}$ (density $5 / 18$ )

Method. The initial charge of a vertex $v$ is 1 if $v \in C$ and 0 otherwise. We then apply some local discharging rules. We shall prove that the final charge of every vertex in $C$ is at least $2 / 9$. This would imply the result.

We set $U=V(G) \backslash C$. Given $X \subseteq V(G)$ and $1 \leq i \leq 9$, we denote by $X_{i}$ (resp. $X_{\geq i}$ ) the set of vertices in $X$ having exactly $i$ vertices (resp. at least $i$ vertices) in their identifier. An $X$-vertex is a vertex in $X$. A vertex is full if its eight neighbours in $\mathcal{G}_{K}$ are in $G$; otherwise it is a side vertex.

We first establish the following properties of $C$.

(i) Two $C_{2}$-vertices are not adjacent.

(ii) Every $C$-vertex has at most one neighbour in $U_{1}$.

(iii) Every full $C_{2}$-vertex has at least three neighbours in $U_{\geq 3}$.

(iv) Every full $C_{3}$-vertex has a neighbour in $U_{\geq 3}$. 
(v) Every $C_{1}$-vertex $(a, b)$ has no neighbour in $U_{1}$ and at most six neighbours in $U_{2}$. Furthermore, if it has six neighbours in $U_{2}$, then either $\{(a-1, b-$ $2),(a-2, b-1),(a+2, b+1),(a+1, b+2)\} \subseteq C$ or $\{(a+1, b-2),(a+$ $2, b-1),(a-2, b+1),(a-1, b+2)\} \subseteq C$.

A defective vertex is a vertex in $C_{1}$ with six neighbours in $U_{2}$. Let $v=(a, b)$ be a defective vertex. The team of $v$ is a set among $\{(a-1, b-2),(a-2, b-$ $1),(a+2, b+1),(a+1, b+2)\}$ and $\{(a+1, b-2),(a+2, b-1),(a-2, b+$ $1),(a-1, b+2)\}$ which is included in $C$. By Property (v), the team exists. Moreover, by Property (i), at least two vertices of the team are in $C_{\geq 3}$. Those vertices are the partners of $v$.

We apply the following discharging rules.

(R1) Every $C$-vertex sends $\frac{2}{9 i}$ to each of its neighbours in $U_{i}$.

(R2) Every defective vertex receives $\frac{1}{54}$ from each of its partners.

Using the above properties, we then prove that the final charge of every vertex $v$ is at least $2 / 9$.

Sketch of proof of Theorem 1.2. We only need to prove that, at the end of the proof of Theorem 1.1, one vertex has final charge greater than $2 / 9$. To do so we shall prove that there is a side $C$-vertex or a $C_{\geq 3}$-vertex and check that such a vertex has final charge at least $\frac{2}{9}+\frac{1}{27}$.

Sketch of proof of Theorem 1.3. Using the Discharging Method, we prove that in average, for every column, there is an extra charge of at least $\frac{4}{81}$ on the three top vertices and an an extra charge of at least $\frac{4}{81}$ on the three top vertices.

Sketch of proof of Theorem 1.5. The $b$ th row of $\mathcal{K}_{k}$ is $R_{b}=\{(a, b) \mid a \in \mathbb{Z}\}$. We have $d\left(C, \mathcal{K}_{k}\right)=\frac{1}{k} \sum_{i=1}^{k} d\left(C, R_{k}\right)$. We show that if $C$ is an identifying code of $\mathcal{K}_{k}(k \geq 3)$, then $d\left(C, R_{1}\right)+d\left(C, R_{2}\right) \geq 1 / 2, d\left(C, R_{k}\right)+d\left(C, R_{k-1}\right) \geq 1 / 2$, $d\left(C, R_{3}\right) \geq 1 / 3$ and $d\left(C, R_{k-2}\right) \geq 1 / 3$. One easily derives that if $C$ is an identifying code of $\mathcal{K}_{5}\left(\right.$ resp. $\left.\mathcal{K}_{6}\right)$, then $d\left(C, \mathcal{K}_{5}\right) \geq 4 / 15 .\left(\right.$ resp. $d\left(C, \mathcal{K}_{6}\right) \leq$ $5 / 18$.

To prove lower bounds on $d^{*}\left(\mathcal{K}_{k}\right)$ for $k \in\{3,4\}$, we use the Discharging Method on the columns $Q_{a}=\{(a, b) \mid 1 \leq b \leq k\}$. Let $C$ be an identifying code of $\mathcal{K}_{k}$. We set the initial charge of every integer $a \in \mathbb{Z}$ to $\operatorname{chrg}_{0}(a)=$ $\left|Q_{a} \cap C\right|$. We say that $a \in \mathbb{Z}$ is satisfied if its charge is least $q_{k}$ and unsatisfied otherwise, where $q_{3}=1$ and $q_{4}=5 / 4$. We apply five discharging rules, Rule $i$ for $i=1$ to 5 one after another. We denote by $\operatorname{chrg}_{i}(a)$ the charge of $a$ after applying Rule $i$.

Rule $i$ : every unsatisfied $a \in \mathbb{Z}$ receives $\min \left\{\operatorname{chrg}_{i-1}(a-i)-q_{k}, q_{k}-\operatorname{chrg}_{i-1}(a)\right\}$ 
from $a-i$, if $a-i$ is satisfied (before Rule $i$ ).

Finally, we prove that, after these rules, every integer $a \in \mathbb{Z}$ is satisfied. This implies $d\left(C, \mathcal{K}_{k}\right) \geq q_{k} / k$.

Acknowledgments This research was supported by FAPERJ, Capes (Project STIC-AmSud 2012), CNPq (Project Universal/Bolsa de Produtividade), ANR (Contract STINT ANR-13-BS02-0007), and the FUNCAP/CNRS project GAIATO INC-0083-00047.01.00/13.

\section{References}

[1] Y. Ben-Haim and S. Litsyn. Exact minimum density of codes identifying vertices in the square grid. SIAM J. Discrete Math. 19: 69-82, 2005.

[2] M. Bouznif, F. Havet, M. Preissman. Minimum-density identifying codes in square grids. AAIM 2016. Lect. Notes Computer Science, 9778: 77-88, 2016.

[3] I. Charon, O. Hudry and A. Lobstein. Identifying Codes with Small Radius in Some Infinite Regular Graphs. Elec. J. Combinatorics 9: R11, 2002.

[4] G. Cohen, S. Gravier, I. Honkala, A. Lobstein, M. Mollard, C. Payan, and G. Zémor. Improved identifying codes for the grid, Comment to [6].

[5] G. Cohen, I. Honkala, A. Lobstein, and G. Zemor. Bounds for Codes Identifying Vertices in the Hexagonal Grid. SIAM J. Discrete Math. 13: 492-504, 2000.

[6] G. Cohen, I. Honkala, A. Lobstein, and G. Zémor. New bounds for codes identifying vertices in graphs. Elec. J. Combinatorics 6(1): R19, 1999.

[7] D.Cranston, G.Yu, A new lower bound on the density of vertex identifying codes for the infinite hexagonal grid. Elec. J. Combinatorics 16: R113, 2009.

[8] A. Cukierman, G. Yu. New bounds on minimum density of an identifying code for the infinite hexagonal graph grid. Disc. App. Math. 161: 2910-2924, 2013.

[9] M. Daniel, S. Gravier, and J. Moncel. Identifying codes in some subgraphs of the square lattice. Theoretical Computer Science 319: 411-421, 2004.

[10] R. Dantas, F. Havet, R. Sampaio. Identifying codes for infinite triangular grids with finite number of rows. Discrete Math., doi 10.1016/j.disc.2017.02.015

[11] M. Jiang. Periodicity of identifying codes in strips. arXiv:1607.03848 [cs.DM]

[12] M. Karpovsky, K. Chakrabarty, and L. B. Levitin. On a new class of codes for identifying vertices in graphs. IEEE Trans. Inform. Theory 44: 599-611, 1998. 